Küchenhoff, Bender, Langner, Lenz-Tönjes:

Effect of Berkson measurement error on parameter estimates in Cox regression models

Sonderforschungsbereich 386, Paper 346 (2003)

Online unter: http://epub.ub.uni-muenchen.de/

Projektpartner
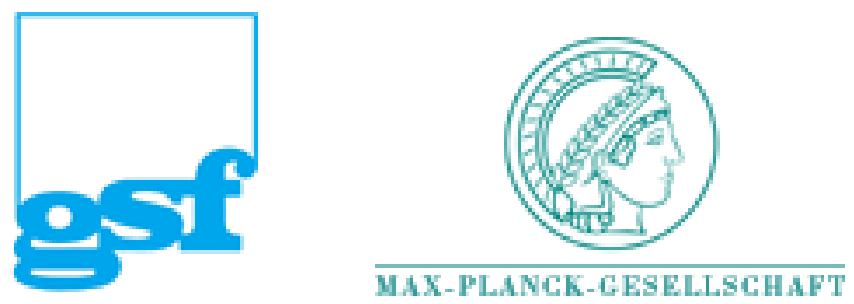


\title{
Effect of Berkson measurement error on parameter estimates in Cox regression models
}

\author{
Helmut Küchenhoff ${ }^{1}$, Ralf Bender ${ }^{2}$, Ingo Langner ${ }^{3}$, Rebecca Lenz-Tönjes ${ }^{3}$ \\ ${ }^{1}$ Department of Statistics, Universität München \\ ${ }^{2}$ Institute of Medical Biometry, Epidemiology \& Informatics, \\ University of Mainz \\ ${ }^{3}$ Department of Epidemiology and Medical Statistics, \\ School of Public Health University of Bielefeld \\ SFB 386 Discussion Paper 346.
}

\begin{abstract}
We study the effect of additive and multiplicative Berkson measurement error in Cox proportional hazard model. By plotting the true and the observed Survivor function and the true and the observed hazard function dependent on the exposure one can get ideas about the effect of this type of error on the estimation of the slope parameter corresponding to the variable measured with error. As an example we analyze the measurement error in the situation of the German Uranium Miners Study both with the graphical methods and with a simulation study. We do not see a substantial bias in the presence of small measurement error and in the rare disease case. Even the effect of a Berkson measurement error with high variance, which is not unrealistic in our example, is a negligible attenuation of the observed effect. This effect is more pronounced for multiplicative measurement error.
\end{abstract}

Keywords: Measurement error, job exposure matrix, Cox model, Berkson error, survival times

\section{Introduction}

In many epidemiological studies exposure measurement can only be conducted with substantial measurement error. For example, in occupational cohort studies, frequently a job-exposure matrix (JEM) is used for exposure assessment rather than individual exposure measurements (Goldberg et al., 1993). A JEM is a cross-classification of occupations and workplace exposures over time. Individual exposures are calculated as time weighted sum of the exposures corresponding to the job history of the considered persons. Examples for epidemiological studies applying a JEM are given by the German Uranium Miners (GUM) Study and the European Study of Cancer Risks among Airline Pilots and Cabin Crew (ESCAPE). In the GUM Study, the risk of lung cancer and other cancers associated with radon exposure is evaluated in a large cohort of uranium miners (Kreuzer et al., 1999). Exposures to radon and its progeny are estimated by using a detailed JEM leading to cumulative radon exposures expressed in working level 
months (WLM). A working level (WL) is defined as $1.3 \times 10^{3} \mathrm{MeV}$ of potential alpha energy per liter of air; 1 WLM equals 1 WL for 170 hours (Kreuzer et al., 2002). In ESCAPE, the individual radiation exposure assessment is based upon individual annual flight hours differentiated in four aircraft categories and a corresponding JEM giving national annual dose rates (in $\mu S v$ per flight hour). The individual cumulative lifetime exposure is calculated as the flight hour weighted sum of aircraft category specific national annual mean doses (Blettner et al., 2003). In both studies, measurement errors of the Berkson type (Carroll et al., 1995) occur, because in the JEM for each job a summary measure such as the annual mean is used as exposure value rather than individual exposure values.

It is well known that the Berkson type error induces no bias in the usual linear model but causes a bias in non-linear models (Carroll et al., 1995). The basic problem of investigating measurement errors within the Cox model is, that the expectation for the observed data in a Cox model with measurement errors in exposures depends on the unknown baseline hazard. This has been discussed by Prentice (1982) for classical additive measurement error. The hazard function cannot be expressed as the product of an arbitrary baseline hazard and a term that depends only on observed data and unknown parameters. Thus, the application of the usual estimation methods for proportional hazards models to Cox models with measurement errors in exposures leads to bias in effect measure estimates. While calssical measurement error has recently received much attention, see e. g. Augustin and Schwartz (2001), Augustin (2003), to our knowledge the effect of Berkson type errors on effect estimation in Cox models has not been systematically analysed. We start our analysis by theoretical considerations calculating the observed dose response relationship in the presence of measurement error. We consider additive as well as multiplicative Berkson measurement error models. To find out the direct effect on parameter estimation we conducted a simulation study. Since our research was motivated by the question of the effect of measurement error in the GUM Study, we mimic the situation there both for our theoretical calculations and for the simulation study.

\section{Theoretical considerations}

We consider a simple survival model with one exposure variable and no further covariates. The true relationship between the exposure $X$ and the response $T$ is given by the survivor function and hazard rate, which are functions dependent on time and exposure.

$$
\begin{array}{r}
P(T \geq t \mid X=x)=S_{\text {true }}(t, x) \\
h_{\text {true }}(t, x)=\left[-\frac{\partial}{\partial t} S_{\text {true }}(t, x)\right] / S_{\text {true }}(t, x)
\end{array}
$$


Typically the exposure response relationship is modelled by the hazard function. The Coxproportional hazard model is given by

$$
\begin{aligned}
h_{\text {true }}(t, x) & =\exp (x \times \beta) \times \lambda_{0}(t) \\
S_{\text {true }}(t, x) & =\exp \left[-\int_{0}^{t} h_{\text {true }}(s, x) d s\right]
\end{aligned}
$$

with baseline hazard $\lambda_{0}(t)$.

In many practical situations we want to make inference about the above true relationship, but we observe the exposure only with measurement error. We denote the error prone observed exposure by $W$. The measurement error is assumed to have Berkson type, which means that the measurement error is independent from the observed value $W$ :

$$
\begin{aligned}
& X=W+U \\
& X=W \times V
\end{aligned}
$$

where $U$ and $V$ are the adiitive and multiplicative Berkson error variables, respectively. The Berkson measurement error typically occurs in situations where $X$ is the individual exposure and $W$ is some kind of mean over exposed individuals like in a JEM.

\subsection{Additive measurement error}

From (1) and (5) we can calculate the observed survivor function assuming that the measurement error is a random variable with density $f_{u}$ and is independent form $w$ and $T$ :

$$
S_{\text {obs }}(t, w):=P(T \geq t \mid W=w)=E\left(S_{\text {true }}(t, x) \mid W=w\right)=\int S_{\text {true }}(t, w+u) f_{u}(u) d u
$$

The observed survivor function $S_{o b s}(t, w)$ is basically an average of the true survivor function $S_{\text {true }}(t, \cdot)$ over a range centered in $w$. If $S_{\text {true }}(t, \cdot)$ is linear in the neighborhood of $w$ the difference between the true and the observed survivor function is small. To assess the effect of measurement error we compare the observed survivor function $S_{o b s}(t, w)$ to the true survivor function $S_{\text {true }}(t, x)$. We consider the simple case of a Cox model with constant baseline hazard $h_{0}=\exp (-2), \beta=1$ and a normally distributed measurement error with expectation 0 and variance $\sigma_{u}^{2}=1$ and calculate the integral in (7) by numerical methods. Note that the survivor function has two arguments, time and exposure. In Fig. 1 we compare $S_{\text {obs }}(t, w)$ with $S_{\text {true }}(t, x)$ as a function of $t$ for fixed $x$, and $w$ respectively (left plot). The relationship between exposure and survival for fixed time $t$ is given in the right plot of Fig. 1. It can be seen that the measurement error makes the survivor function flatter. Although this is similar to other regression models direct calculations for the bias when estimating $\beta$ without ignoring the measurement error is much more difficult than in a standard regression context (see e. g. Küchenhoff and 
Carroll, 1997). To get an idea of this bias we explore the relationship between $X$ and $T$ by analyzing the hazard rate. The observed hazard rate is given by, see (2)

$$
h_{o b s}(t, w)=\frac{\partial}{\partial t} S_{o b s}(t, w) / S_{o b s}(t, w) .
$$

Note, that $h_{o b s}(t, w)$ cannot be derived by integration of hazard function due to the definition of the hazard as a conditional probability, see Pepe et al. (1989). In the Cox model and in many parametric survival models the hazard has a log linear dependence on $X$, resulting in a straight line on a logarithmic plot. In Fig. 2 the relationship between the hazard and the true $x$, rep. the observed $w$, i.e. the functions $h_{o b s}(t, \cdot)$ and $h_{\text {true }}(t, \cdot)$, are plotted for fixed values of t. We use the simple Cox model with the parameters form Fig. 1 The left plot of Fig. 2 shows that the relationship is no longer log linear and an attenuation effect can be seen which results in a downward bias in the estimation of $\beta$. In the right plot of Fig. 2 we choose an earlier time point $(\mathrm{t}=0.1)$ which results in a higher survival and can be regarded as the case where the rare disease assumption can be justified. There the hazard can be well approximated by

$$
h_{\text {obs }}(t, w)=\int h_{\text {true }}(t, w+u) f_{u}(u) d u=\exp \left(w \beta+\sigma_{u}^{2} / 2\right) .
$$

This results in a relationship between $W$ and the hazard which is still log linear with the same slope. So in that case no bias in the estimation of the effects can be expected, as also can be seen from Fig. 2, right plot.

We are also interested in the effect of measurement error on the shape of the observed hazard function. In Fig. 3 we compare the function $h_{\text {true }}(\cdot, x)$ and $h_{\text {obs }}(\cdot, w)$ for different fixed values of $x$ and $w$. It can be seen that the observed hazard function differs in shape from the constant true hazard function. Comparing the two observed hazard function, we see that the proportional hazard assumption does not hold in the case of measurement error. So ignoring measurement error could lead to an inconsistent estimation of the baseline hazard and incorrect tests for the assumption of proportional hazards.

\subsection{Multiplicative measurement error}

We now explore the effect of a multiplicative Berkson error. There, we derive from (6)

$$
\begin{aligned}
S_{o b s}(t, w) & :=P(T \geq t \mid W=w)=E\left(S_{\text {true }}(t, x) \mid W=w\right)=\int S_{\text {true }}(t, w \times v) f_{v}(v) d v \\
h_{o b s}(t, w) & =\frac{\partial}{\partial t} S_{o b s}(t, x) / S_{o b s}(t, x) .
\end{aligned}
$$

We assume a multiplicative lognormal measurement error with $E(v)=1$ and $\operatorname{Var}(v)=0.5^{2}$. The sizes of additive and multiplicative measurement error are not directly comparable due to the different structure. In our calculation the additive measurement error has the same variance 
as the multiplicative measurement error when $x=2$. In Fig. 4 the observed and the true hazard function are compared. The plot on the left side shows an increasing effect of the measurement error for higher values of the exposure. Here the attenuation in estimating $\beta$ could be substantial. In Fig. 4 it can be seen that the effect does not vanish for the rare disease case. Here, even small upward bias could occur, since the observed curve has a bigger slope when $x$ is varying between 0 and 2. Concerning the dependence of the hazard function as a function of time indicates the violation of the proportional hazard assumption and the clear effect of measurement error on the hazard function $h_{0}(., x)$ (plot not shown).

\subsection{Application to the GUM study}

We turn to the situation of the GUM Study. We consider a Cox proportional hazards model with exposure and age at baseline as covariates. We use the Gompertz distribution (Elandt-Johnson and Johnson, 1980) to describe mortality in a realistic way. This leads to the Cox-Gompertz model

$$
\begin{aligned}
h(t) & =h_{0}(t) \times \exp \left(\beta_{\text {age }} \times \text { age }+\beta_{\text {radon }} \times \text { radon }\right) \\
h_{0}(t) & =\lambda \times \exp (\alpha \times t)
\end{aligned}
$$

For our calculation we use $\lambda=7 \times 10^{-8}, \alpha=0.2138, \beta_{\text {age }}=0.15$ and $\beta_{\text {radon }}=0.001$. With these parameters we get a mean life expectancy of 66.86 years, which is the correct value for German men in 1962 and an expected number of deaths which correspond to the observed number of deaths in the GUM Study. We assume a radon effect of $\beta_{\text {radon }}=0.001$ for the cumulative radon exposure measured in WLM, which corresponds to a hazard ratio (HR) of $H R=1.65$ per 500 WLM. This effect lies in the lower area of possible health effects of radon proposed by the National Research Council (1999). For theoretical evaluations we consider a fixed value for age at baseline of 30 years. For the additive Berkson error we consider $\sigma=359$ and $\sigma=1016$. Since the observed standard deviation in the study is 508 , measurement error variance is related to the observed variance by the factor 0.5 and 4 . These measurement errors are rather high, but not unrealistic in this study. We also explore the effect of multiplicative measurement error. Here we assume standard deviations of 0.34 and 1.16. In Fig. 5 we display the effect of the additive measurement error (left plot) and multiplicative measurement error (right plot) on the survivor function. The influence of measurement on estimating the slope parameter $\beta$ is explored in Fig. 6. The attenuation effect can be seen in the presence of higher exposure values. For the multiplicative Berkson error the effect for high exposure is covered. So the slope parameter is biased downwards in this setting. 


\section{Simulation study}

To get an impression of the amount of possible bias in epidemiological studies applying a JEM, we performed a simulation study based upon the baseline data of the GUM Study (Kreuzer et al., 2002). In the simulations, the variables entry date, age at entry (mean 24.3, SD 8.4 years) and the cumulative radon exposure (mean 266.8, SD 507.8 WLM) are used for a sample of $n=59813$ male uranium miners with birth date since 01.01.1900 and entry date between 1946 and 1989 . To simulate survival times, we used the Cox-Gompertz model $(12,13)$ with the same parameters as described in Section 2, i.e. $\lambda=7 \times 10^{-8}, \alpha=0.2138, \beta_{\text {age }}=0.15$ and $\beta_{\text {radon }}=0.001$. To evaluate the effect of measurement error in the Cox model via simulations, the effect of the covariates has to be translated from hazards to survival times, because the usual software packages for Cox models require the individual survival time data, not the hazard function. It can be shown that survival times for the Cox-Gompertz model $(12,13)$ can be expressed as

$$
T=\frac{1}{\alpha} \log \left[1-\frac{\alpha \times \log (Z)}{\lambda \times \exp \left(\beta_{\text {age }} \times+\beta_{\text {radon }} \times \text { radon }\right)}\right]
$$

where $Z$ is a random variable with uniform distribution on [0;1], (Bender et al., 2003). Thus, equation (14) can be used to simulate appropriate survival times in the considered situation. We generated and analysed data for the additive Berkson error model (5) by using the following steps.

1. For each individual generate a normally distributed error $U$ with mean 0 and variance $\sigma^{2}$

2. Add the Berkson error $U$ to the observed radon exposure $R_{o b s}: R_{t r u e}=R_{o b s}+U$

3. For each individual generate a value sampled from a uniform distribution on the interval $[0,1]$

4. Use transformation (14) with radon $=R_{\text {true }}$ to get Gompertz distributed survival times

5. Use the date 31.12.1998 (end of follow-up in the GUM Study) for censoring

6. Estimate the parameters $\beta_{\text {age }}$ and $\beta_{\text {radon }}$ by using both $R_{\text {true }}$ and $R_{o b s}$

7. Evaluate the bias induced by measurement error by comparison with $\beta_{\text {radon }}$.

For generating normally and uniformly distributed random numbers, the SAS (1985) functions NORMAL and UNIFORM were used, respectively. For parameter estimation of the Cox models the SAS (1991) procedure PHREG was applied. The steps 1-7 were repeated for different values of $\sigma^{2}$ varying from $1 \%$ to $800 \%$ of the observed variance of $R_{o b s}$.

For the multiplicative Berkson error model (6) the first two steps were replaced by 
1. For each individual generate a lognormally distributed error $V$ with mean 1 and variance $\sigma^{2}$.

2. Multiply the Berkson error by the observed radon exposure : $R_{\text {true }}=R_{o b s} \times V$

The steps 1-7 for the multiplicative Berkson error model were repeated for different values of $\sigma^{2}$ such that the variance of $R_{\text {true }}$ ranges between $110 \%$ and $250 \%$ of the variance of $R_{o b s}$. For each measurement error model and each value of $\sigma^{2} 1000$ simulations are performed.

To describe the results of the simulation we use the mean percentage bias. It is defined by

$$
M P E=\frac{\overline{\hat{\theta}}-\theta}{\theta} \times 100 .
$$

with $\theta$ being the considered regression coefficient in the Cox-Gompertz-model ( $\beta_{\text {age }}$ or $\beta_{\text {radon }}$ ) and $\overline{\hat{\theta}}$ being the mean in the 1000 simulations. The same measures are used to evaluate the quality of standard errors (SEs). Because the true value (standard deviation of parameter estimator) is unknown, the empirical standard deviation of 1000 estimated regression coefficients is used for $\theta$ in this case.

The MPE values of the simulations regarding the additive Berkson error model (5) are shown in Table 1. We can conclude that there is a dilution bias induced by Berkson errors for all variables, even for age which is measured without error. Interestingly, there is a small dilution bias even if we use the exposure values $R_{\text {true }}$, i.e. the exposure without error, for estimation. A severe bias $(M P E<-10 \%)$ is observed only for quite large error variances $\left(\sigma^{2}>100 \%\right)$ of the variance of $R_{o b s}$. The same holds for the adequacy of the standard errors. A considerable underestimation of the SEs is only found for quite large error variances (Table 2).

The MPE values of the simulations regarding the multiplicative Berkson error model (6) can be found in Table 3. Again, we observe a dilution bias. However, the bias seems to be more pronounced than in the case of additive errors. There is a different attenuation effect between the variables measured with (radon) and without error (age). Now, we observe a considerable underestimation of SEs, but only for the covariate measured with error (Table 4).

\section{Discussion}

We performed two ways of exploring the effect of Berkson measurement error models in the Cox model. First, we gave theoretical considerations on the observed exposure response relationship. The observed survival curve can be calculated by integration assuming a Berkson type of error. The observed hazard can be deduced from the observed survival. Since the Cox model is characterized by the log linear exposure hazard relationship for every fixed point of time, the logarithmic plot of the hazard as a function of the observed exposure gives interesting 
insights on the effect of measurement error in different settings. Our findings show that the observed exposure hazard relationship is not log linear in the presence of high multiplicative or additive measurement error. In most situations measurement error causes a flattening of the relationship between the observed exposure and the hazard compared to the true exposure hazard relationship. This is only true for a higher risk, while for a high probability of survival the effect of measurement error is low, which is in similar to the results of Prentice (1982) for additive measurement error.

Since direct theoretical calculations for the bias of the effect estimates are not feasible in this complex context we performed a simulation study to quantify this bias. The simulation study design is close to the situation of the GUM study. We find a downward bias of the effect estimates dependent on the measurement error variance. This bias is only relevant for high additive measurement error or multiplicative measurement error. The results of the simulation study are in accordance to the plots resulting from the theoretical calculations. Furthermore, our simulations show an attenuation effect of measurement error on other covariates (age in the situation of our simulation design). We also performed simulations for heteroscedastic additive Berkson measurement error which yielded similar results similar as in the homoscedastic case.

Another relevant issue in the Cox model is the estimation of the baseline hazard. This estimation is based on the proportional hazard assumption. Our calculations show that the observed hazard function as a function of time $t$ differs substantially in shape from the true hazard function. The shape depends on the observed exposure, indicating that the proportional hazard assumption does not hold for the observed data.

In summary the effect of Berkson measurement error in the Cox model should be taken into account for the estimation in the presence of multiplicative or high additive measurement error in the exposure when the rare disease assumption is violated. In that cases the effect estimates are attenuated for the exposure and also for further covariates without measurement error. Since the log linear exposure hazard relationship and the proportional hazard assumption do not hold, one fits a wrong model for the data when the measurement error is ignored. Although our plots give only results for special situations, one gets an idea of the effect of Berkson measurement error. More calculations are useful especially for different types of true hazard functions. Note that we only considered Berkson measurement error which is known to cause much smaller effects on effect estimation than a classical additive or multiplicative measurement error. Our results have shown that the development of methods to adjust for Berkson errors would be useful. Such methods could also be applied in the case of additive measurement error when the regression calibration method (Carrroll et al.,1995) is used for correction of measurement error. There, basically a Berkson component of measurement error is still not taken into account. 


\section{References}

[1] Augustin, T. (2003): An exact corrected log-likelihood function for Cox's proportional hazards model. Scandinavian Journal of Statistics, in press.

[2] Augustin, T., Schwarz, R. (2002): Cox's proportional hazards model under covariate measurement error. A review and comparison of methods. In: Van Huffel, S. and Lemmerling, P. (eds): Total least squares and Error-in-Variables Modelling: Analysis, Algorithms and Applications, Kulwer; Dortrecht, 175-184.

[3] Bender, R., Augustin, T., Blettner, M. (2003): Generating survival times to simulate Cox proportional hazards models. Discussion Paper Nr. 338, SFB 386, Munich.

[4] Blettner, M., Zeeb, H., Auvinen, A., Ballard, T.J., Caldora, M., Eliasch, H., Gundestrup, M., Haldorsen, T., Hammar, N., Hammer, G.P., Irvine, D., Langner, I., Paridou, A., Pukkala, E., Rafnsson, V., Storm, H., Tulinius, H., Tveten, U., Tzonou, A. (2003). Mortality from cancer and other causes of male airline cockpit crew in Europe. International Journal of Cancer 106 (in press).

[5] Carroll, R.J., Ruppert, D., Stefanski, L.A. (1995). Measurement Error in Nonlinear Models. Chapman and Hall, London.

[6] Elandt-Johnson, R.C.,Johnson, N.L. (1980): Survival Models and Data Analysis. Wiley, New York.

[7] Goldberg, M., Kromhout, H., Guénel, P., Fletcher, A.C., Gérin, M., Glass, D.C., Heederik, D., Kauppinen, T., Ponti, A. (1993): Job exposure matrices in industry. International Journal of Epidemiol. 22 (Suppl. 2), 10-15.

[8] Kreuzer, M., Grosche, B., Brachner, A., Martignoni, K., Schnelzer, M., Schopka, H.-J., Brüske-Hohlfeld, I., Wichmann, H.-E. and Burkart, W. (1999): The German Uranium Miners Cohort Study: Feasibility and first results. Radiation Res. 153 (Suppl.), 56-58.

[9] Kreuzer, M., Brachner, A., Lehmann, F., Martignoni, K., Wichmann, H.E., Grosche, B. (2002): Characteristics of the German Uranium Miners Cohort Study. Health Physics 83, 26-34.

[10] Küchenhoff, H. and Carroll, R.-J. (1997): Segmented Regression with errors in predictors: Semi-parametric and parametric methods.Statistics in Medicine 16,169-188.

[11] Lee, E. T. (1992): Statistical Methods for Survival Data Analysis. Wiley, New York. 
[12] National Research Council (1999): Committee on Health Risks of Exposure to Radon (BEIR VI): Health Effects of Exposure to Radon. National Academy Press, Washington, D.C.

[13] Pepe, M. S., Self, M. S. and Prentice, R.L. (1989): Further results in covariate measurement errrors in cohort with time to respones. Statistics in Medicine 8, 829-838.

[14] Prentice, R. L. (1982): Covariate measurement errors and parameter estimation in a failure time regression model. Biometrika 69, 331-342.

[15] SAS (1991): SAS Language Guide for Personal Computers, Version 6 Edition. SAS Institute Inc., Cary, NC.

\section{Acknowledgements}

Supported by part by German Research foundation (DFG) grants BE 2485/1-1 and SFB 386. WE thank Bernd Grosche, Michaela Kreuzer and Anemarie Brachner for providing the data of the German Uranium Miners (GUM) Study. 


\section{Tables}

Table 1: Mean percent error (MPE) of the parameter estimates in the Cox-Gompertz model by using the exposure without $\left(R_{\text {true }}\right)$ and with additive Berkson error with variance $\sigma^{2}\left(R_{o b s}\right)$

\begin{tabular}{|rrrrrr|}
\hline \multicolumn{1}{c}{$\sigma$} & \multicolumn{2}{c}{ Var $(U)$} & \multicolumn{2}{c|}{ Age } \\
& $\sqrt{\operatorname{Var}\left(R_{\text {obs }}\right)}$ & $R_{\text {true }}$ & $R_{\text {obs }}$ & $R_{\text {true }}$ & $R_{\text {obs }}$ \\
50.8 & $1 \%$ & -1.78 & -1.90 & -0.62 & -0.73 \\
113.6 & $5 \%$ & -1.76 & -2.32 & -0.60 & -1.17 \\
160.6 & $10 \%$ & -1.76 & -2.89 & -0.64 & -1.75 \\
227.1 & $20 \%$ & -1.62 & -3.84 & -0.66 & -2.85 \\
278.2 & $30 \%$ & -1.56 & -4.86 & -0.62 & -3.86 \\
321.2 & $40 \%$ & -1.57 & -5.88 & -0.62 & -4.84 \\
359.1 & $50 \%$ & -1.46 & -6.74 & -0.58 & -5.78 \\
439.8 & $75 \%$ & -1.33 & -8.87 & -0.60 & -8.06 \\
507.8 & $100 \%$ & -1.26 & -10.91 & -0.64 & -10.15 \\
718.2 & $200 \%$ & -0.93 & -17.51 & -0.53 & -16.90 \\
879.6 & $300 \%$ & -0.79 & -22.66 & -0.54 & -22.19 \\
1015.6 & $400 \%$ & -0.63 & -26.91 & -0.53 & -26.46 \\
1436.3 & $800 \%$ & -0.37 & -38.51 & -0.50 & -37.88 \\
\hline
\end{tabular}


Table 2: Mean percent error (MPE) of the standard errors parameter in the Cox-Gompertz model by using the exposure without (R_true) and with additive Berkson error with variance $\sigma^{2}\left(R_{o b s}\right)$

\begin{tabular}{|rrrrrr|}
\hline \multirow{2}{*}{$\sigma$} & \multirow{2}{*}{$\operatorname{Var}(U)$} & \multicolumn{2}{c|}{ Exposure } & \multicolumn{2}{c|}{ Age } \\
& $\operatorname{Var}\left(R_{\text {obs }}\right)$ & $R_{\text {true }}$ & $R_{\text {obs }}$ & $R_{\text {true }}$ & $R_{\text {obs }}$ \\
50.8 & $1 \%$ & -0.13 & -0.67 & -1.46 & -1.48 \\
113.6 & $5 \%$ & -3.91 & -4.57 & +2.03 & +1.98 \\
160.6 & $10 \%$ & -2.59 & -3.09 & -2.47 & -2.86 \\
227.1 & $20 \%$ & -4.64 & -5.91 & -3.42 & -4.45 \\
278.2 & $30 \%$ & +3.34 & -1.23 & +1.07 & -0.55 \\
321.2 & $40 \%$ & -2.16 & -5.52 & -2.12 & -4.33 \\
359.1 & $50 \%$ & +0.48 & -1.36 & -2.66 & -2.63 \\
439.8 & $75 \%$ & -1.86 & -7.55 & 1.40 & -2.23 \\
507.8 & $100 \%$ & +1.76 & -6.94 & -2.63 & -7.30 \\
718.2 & $200 \%$ & -0.64 & -8.17 & 0.35 & -4.93 \\
879.6 & $300 \%$ & -3.36 & -12.80 & -3.60 & -12.26 \\
1015.6 & $400 \%$ & -0.20 & -13.88 & -0.88 & -11.37 \\
1436.3 & $800 \%$ & +1.44 & -14.46 & -4.90 & -8.66 \\
\hline
\end{tabular}


Table 3: Mean percent error (MPE) of the parameter estimates in the Cox-Gompertz model by using the exposure without $\left(R_{\text {true }}\right)$ and with multiplicative Berkson error with variance $\sigma^{2}$ $\left(R_{o b s}\right)$

\begin{tabular}{|crrrrr|}
\hline \multirow{2}{*}{$\sigma$} & \multicolumn{2}{c}{$\operatorname{Var}(U)$} & \multicolumn{2}{c|}{ Exposure } & \multicolumn{2}{c|}{ Age } \\
& $\frac{\operatorname{Var}\left(R_{\text {obs }}\right)}{}$ & $R_{\text {true }}$ & $R_{\text {obs }}$ & $R_{\text {true }}$ & $R_{\text {obs }}$ \\
0.309 & $110 \%$ & -1.51 & -10.39 & -0.61 & -3.45 \\
0.512 & $130 \%$ & -1.11 & -20.20 & -0.59 & -6.53 \\
0.637 & $150 \%$ & -0.98 & -26.19 & -0.58 & -8.24 \\
0.833 & $200 \%$ & -0.79 & -34.98 & -0.55 & -10.35 \\
0.957 & $250 \%$ & -0.71 & -40.08 & -0.54 & -11.32 \\
\hline
\end{tabular}

Table 4: Mean percent error (MPE) of the parameter estimates in the Cox-Gompertz model by using the exposure without $\left(R_{\text {true }}\right)$ and with additive Berkson error with variance $\sigma^{2}\left(R_{o b s}\right)$

\begin{tabular}{|crrrrr|}
\hline \multirow{2}{*}{$\sigma$} & \multicolumn{2}{c}{$\operatorname{Var}(U)$} & \multicolumn{2}{c|}{ Exposure } & \multicolumn{2}{c|}{ Age } \\
& $\frac{\operatorname{Var}\left(R_{\text {obs }}\right)}{}$ & $R_{\text {true }}$ & $R_{\text {obs }}$ & $R_{\text {true }}$ & $R_{\text {obs }}$ \\
0.309 & $110 \%$ & +0.28 & -14.73 & -0.52 & -1.97 \\
0.512 & $130 \%$ & +4.19 & -20.74 & +2.71 & -2.00 \\
0.637 & $150 \%$ & -0.17 & -23.37 & -1.22 & -1.62 \\
0.833 & $200 \%$ & -0.97 & -21.44 & -2.65 & -3.82 \\
0.957 & $250 \%$ & +4.71 & -23.53 & +1.55 & +3.35 \\
\hline
\end{tabular}




\section{Figures}
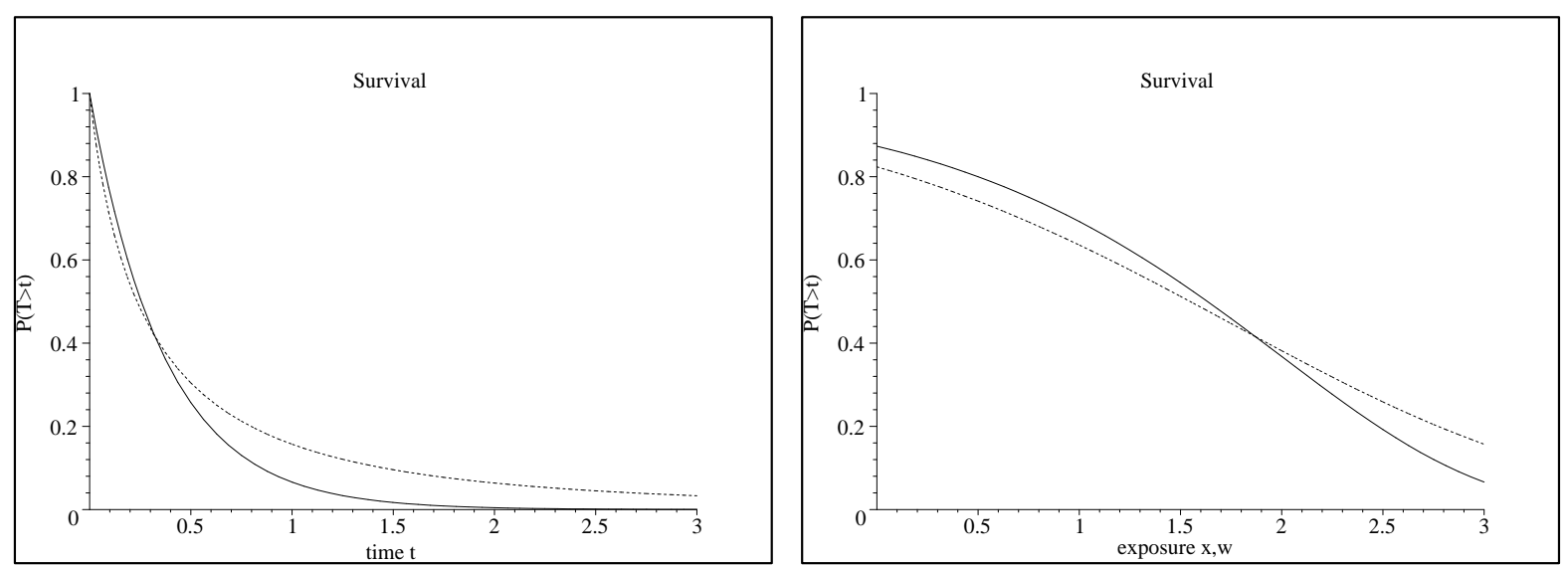

Figure 1: Comparison of the observed survivor function $S_{o b s}(t, \cdot)$ (dashed line) and the true survivor function $S_{\text {true }}(t, \cdot)$ (solid line). We assume a Cox model with constant baseline hazard $h_{0}=\exp (-2), \beta=1$ and a standard normal additive Berkson measurement error. In the left plot the exposure is fixed being 1 , while in the right plot time $\mathrm{t}=1$.
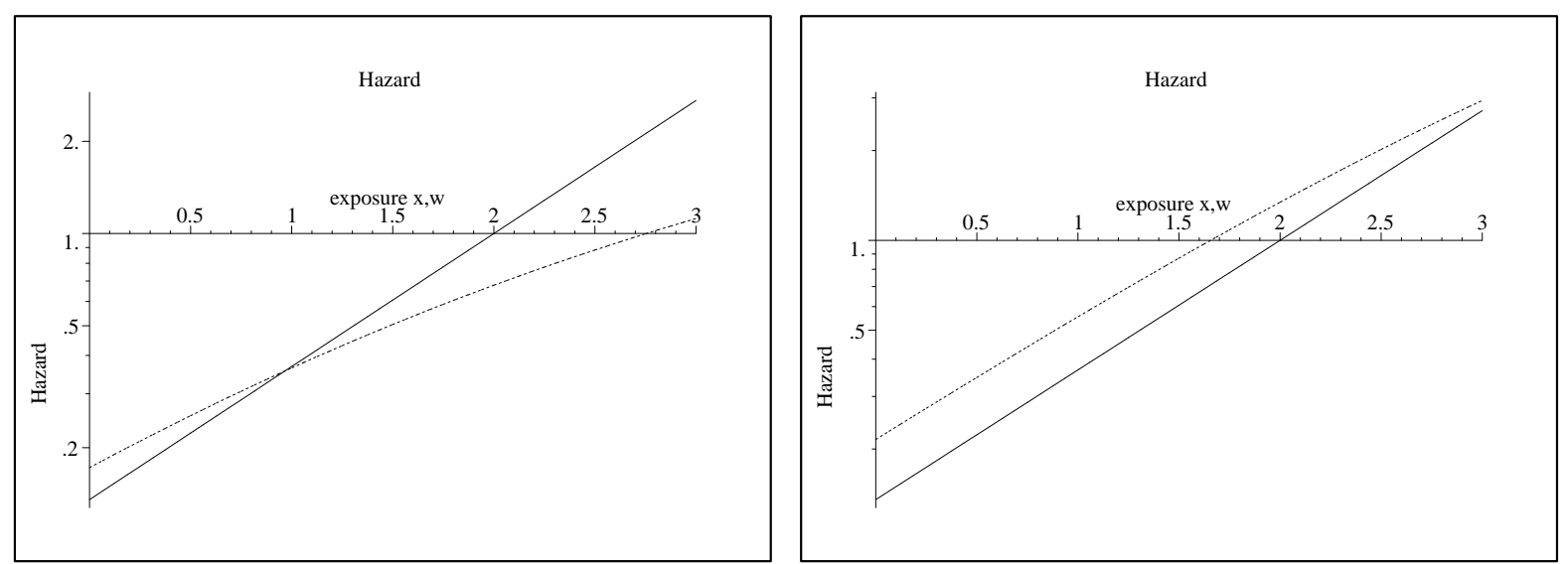

Figure 2: Comparison of the observed hazard $h_{o b s}(t, \cdot)\left(\right.$ dashed line) and the true hazard $h_{\text {true }}(t, \cdot)$ (solid line). We assume a Cox model with constant baseline hazard $h_{0}=\exp (-2), \beta=1$ and a standard normal additive measurement error. In the left plot we choose $t=1$, while in the right plot $\mathrm{t}=0.1$ (rare disease assumption). 

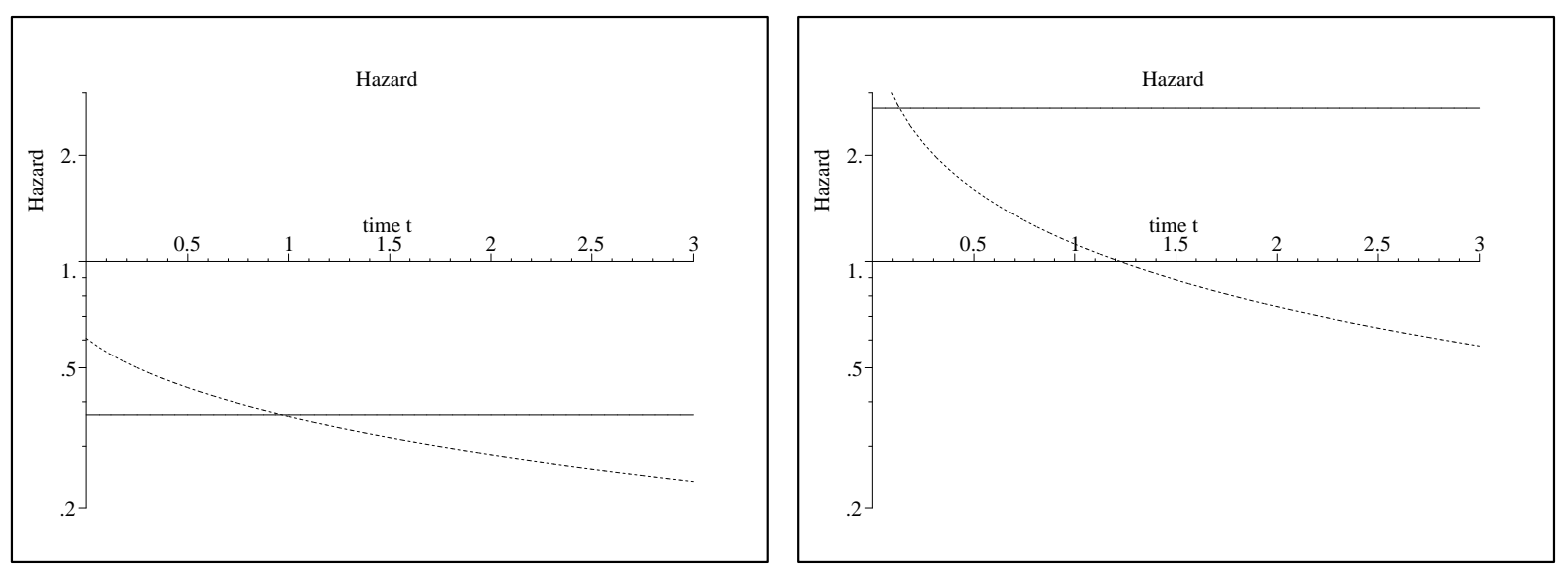

Figure 3: Comparison of the observed hazard function $h_{o b s}(\cdot, w)$ (dashed line) and the true hazard function $h_{\text {true }}(\cdot, x)$ (solid line) as a function of time for the Cox model with constant baseline hazard and $\beta=1$ with statndard normal measurement error. The left plot gives the result for fixed $\mathrm{x}=1, \mathrm{w}=1$, and in the right plot we fix $\mathrm{x}=3, \mathrm{w}=3$.
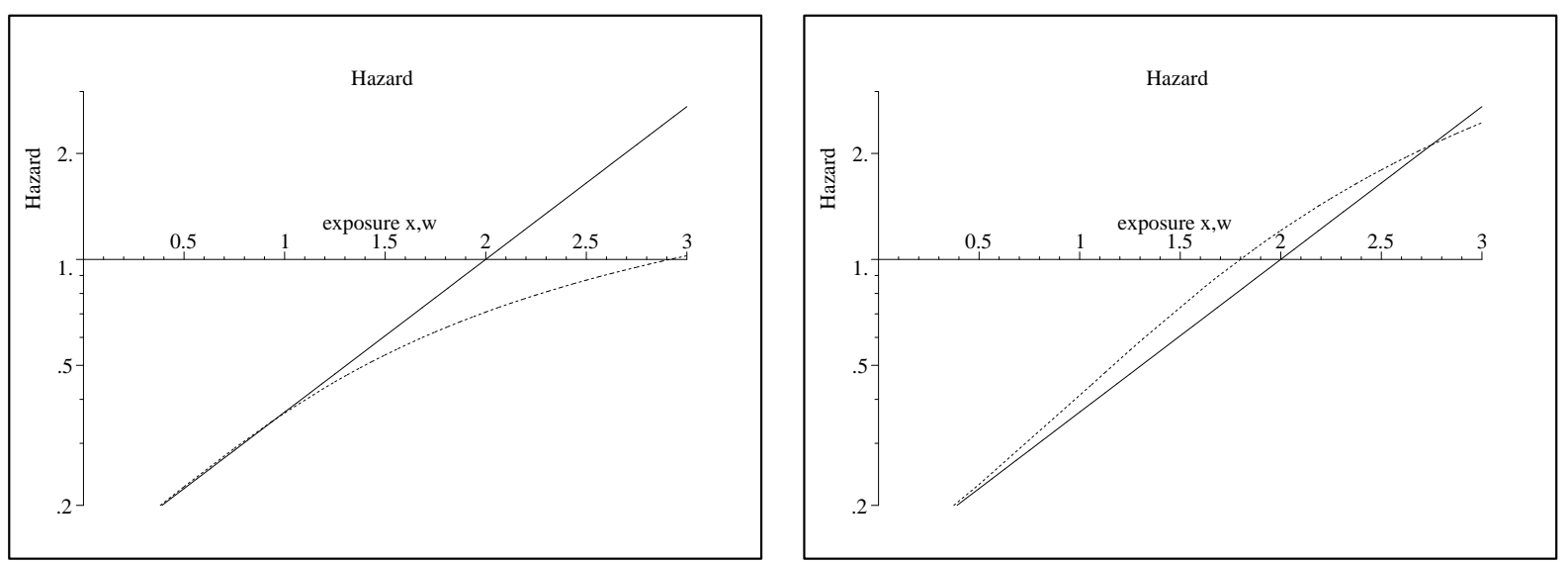

Figure 4: Comparison of the observed hazard $h_{o b s}(t, \cdot)$ (dashed line) and the true hazard $h_{\text {true }}(t, \cdot)$ (solid line). We assume a Cox model with constant baseline hazard $h_{0}=\exp (-2)$, $\beta=1$ and a log normal multiplicative measurement error with standard deviation 0.5.In left plot, $t=1$ and in the left plot $t=0.1$, which reflects the rare disease assumption. 

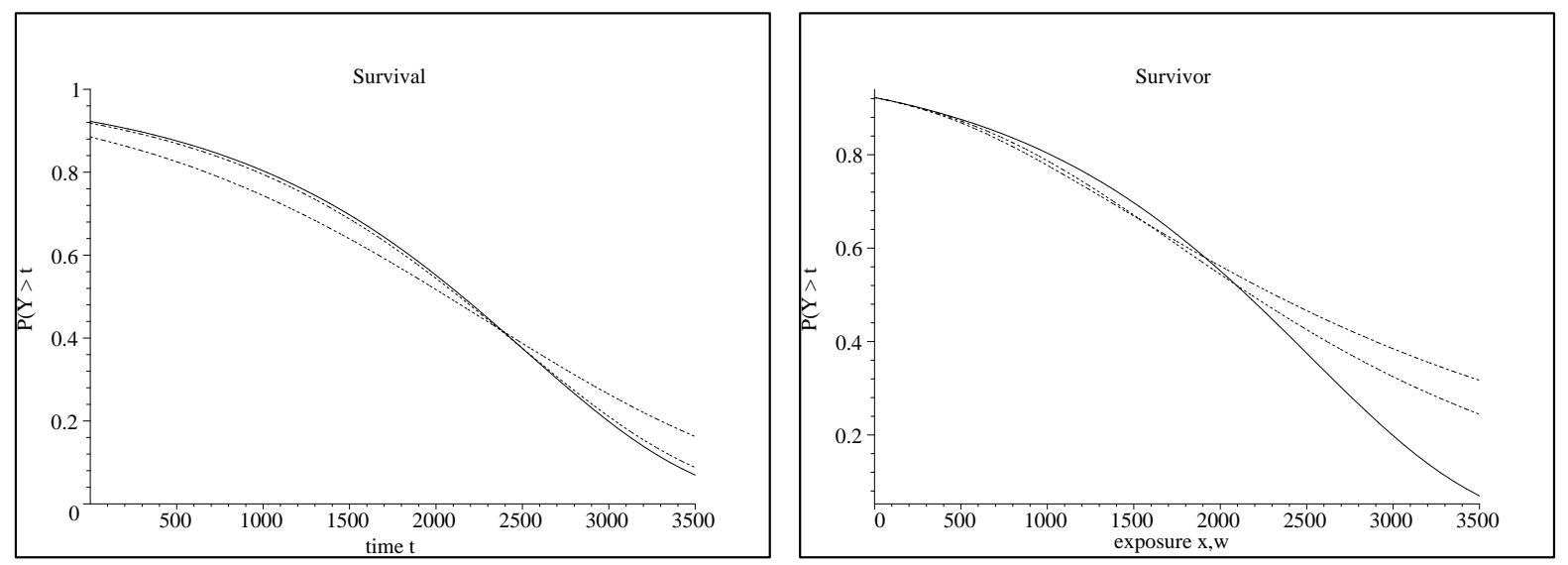

Figure 5: Comparison of the observed survivor function $S_{o b s}(t, \cdot)$ (dashed lines) (left plot) and the true survivor function $S_{\text {true }}(t, \cdot)$ (solid line). We assume two different amounts of additive Berkson error(left plot) and two different amounts of multiplicative Berkson error (right plot) for fixed $\mathrm{t}=30$. We assume a Cox model with parameters motivated by the German Uranium miners (GUM) Study.
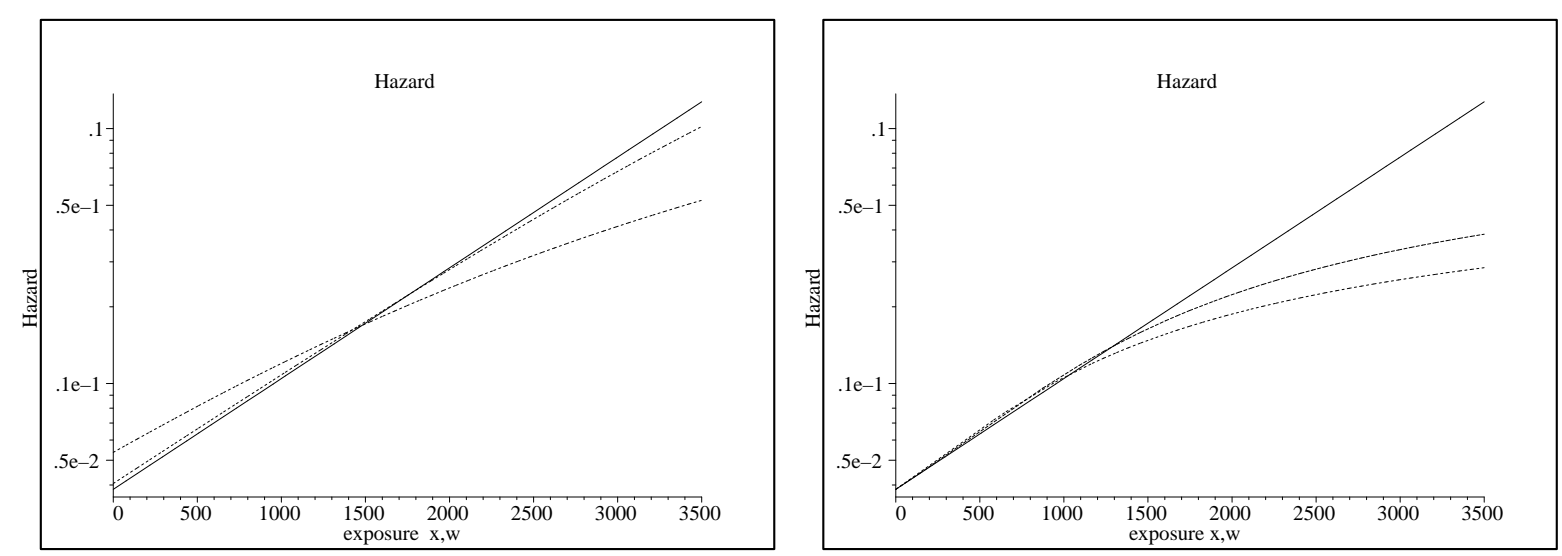

Figure 6: Comparison of the observed hazard $h_{o b s}(t, \cdot)$ (dashed lines) and the true hazard function $h_{\text {true }}(t, \cdot)$ (solid line). We assume two different amounts of additive Berkson error(left plot) and two different amounts of multiplicative Berkson error (right plot) for fixed $t=30$. We assume a Cox model with parameters motivated by the German Uranium miners (GUM) Study. 